\title{
Duchenne Muscular Dystrophy (DMD) with Severe Cardiomyopathy
}

\author{
Ji Hyoung Park ${ }^{1, *}$, Kwang Ho Lee ${ }^{2}$, Wi Kwang Wang ${ }^{3}$ and Hyun Kyo Lim* \\ ${ }^{1}$ Department of Anesthesiology and Pain Medicine, Yonsei University Wonju College of Medicine \\ 20 Ilsan-ro, Wonju, Korea \\ ${ }^{2}$ Department of Anesthesiology and Pain Medicine, Yonsei University Wonju College of Medicine \\ 20 Ilsan-ro, Wonju, Korea \\ ${ }^{3}$ Department of Anesthesiology and Pain Medicine, Yonsei University Wonju College of Medicine \\ 20 Ilsan-ro, Wonju, Korea \\ *'Department of Anesthesiology and Pain Medicine, Yonsei University Wonju College of Medicine \\ 20 Ilsan-ro, Wonju, Korea \\ *Corresponding author's email: killerjhjh [AT] yonsei.ac.kr
}

\begin{abstract}
Duchenne muscular dystrophy (DMD) is a progressive myopathy. The development of respiratory therapy has increased the life expectancy of DMD patients. This change has increased the chances of anesthesia administration in DMD patients with advanced cardiomyopathy. We report a severe cardiomyopathy case in a 14-yearold boy with DMD, adrenal insufficiency, and severe mental retardation, who experienced a sudden cardiac arrest with successful resuscitation. The patient underwent feeding gastrostomy surgery to relieve recurrent aspiration pneumonia, during which cardiac index and heart rate decreased. Cardiomyopathy has emerged as a new challenge in DMD patients; it is important to maintain end organ perfusion by proper function of the left ventricle.
\end{abstract}

Keywords--- Cardiomyopathy, Duchenne muscular dystrophy, End organ perfusion

\section{INTRODUCTION}

Duchenne muscular dystrophy (DMD) is an X-linked recessive disorder caused by mutation of the dystrophin gene [1]. DMD is a progressive muscle wasting disorder that affects skeletal muscle, myocardium, respiratory, and bulbar muscles.

The life expectancy of DMD patients has gradually increased to 30-35 years of age. This prolongation of survival is due to improved treatment and long-term care with the use of assisted noninvasive positive ventilation, glucocorticoid therapy, cardiomyopathy management, and nutritional support [2]. Prolonged life expectancy in DMD patients may increase the chances of receiving anesthesia with advanced disease status, which is a major challenge for anesthesiologists.

Progressive dysphagia is a common complication in older DMD survivors, and feeding gastrostomy is necessary to prevent aspiration and provide nutrition [3]. Development of advanced respiratory therapy has improved respiratory care for DMD patients, making cardiomyopathy the leading cause of death in this population. Cardiomyopathy increases the risk of anesthetic complications in patients with DMD. Therefore, before surgery, a detailed assessment should be performed, and complete monitoring to evaluate cardiac output and aggressive treatment is necessary intraoperatively [4].

\section{CASE}

A 14-year-old patient with DMD, adrenal insufficiency, and severe mental retardation was admitted for fever, cough, sputum, and vomiting. Asystole occurred during hospitalization due to worsening pneumonia. After successful CPR, transthoracic echocardiography (TTE) was performed and revealed enlarged four chambers with an ejection fraction of $20 \%$ (LVEDD/BSA $\left.4.1 \mathrm{~cm} / \mathrm{m}^{2}\right)$. Dilated cardiomyopathy was diagnosed, and norepinephrine $(0.05 \mathrm{mcg} / \mathrm{kg} / \mathrm{min})$ and dopamine $(5 \mathrm{mcg} / \mathrm{kg} / \mathrm{min})$ therapy was initiated. Solondo and florinef were replaced with intravenous injection of hydrocortisone $\left(100 \mathrm{mg} / \mathrm{BSA} / \mathrm{m}^{2}\right)$. Inotropic treatment was discontinued on the $7^{\text {th }}$ day of admission, and follow-up TTE 
revealed an increased ejection fraction up to $31 \%$. Respiratory support was performed, but pneumonia persisted with repeated aspiration of oral secretions. A swallowing test showed inability to ingest orally, so we decided to perform the feeding gastrostomy under general anesthesia.

When the patient arrived in the operating room, a central venous catheter (Arrow double lumen catheter) was secured on the right subclavian vein. Standard monitoring was initiated including five-lead EKG, pulse oximetry, and noninvasive blood pressure monitoring. Defibrillation patches were attached, and preoxygenation was started. Midazolam $2.5 \mathrm{mg}$, sufentanil $75 \mathrm{mcg}$, and rocuronium $25 \mathrm{mg}$ were intravenously injected to minimize myocardial depression. After endotracheal intubation, anesthesia was maintained with total intravenous anesthesia using propofol and remifentanil. Bispectral index (BIS) monitoring was used, and its score was maintained between 40 and 60 . The radial artery pulse was not palpable, so arterial cannulation was performed at the right femoral artery under ultrasound guidance.

During operation, non-invasive cardiac output monitoring (FloTrac/Vigileo Edward) was used. The patient's heart rate ranged from 70 to 90 beats/minute (BPM), and the cardiac index remained above $2 \mathrm{~L} / \mathrm{min} / \mathrm{m}^{2}$. The operation proceeded for 20 minutes without problem. However, at the end of the operation, the cardiac index decreased from 3.2 to $1.7 \mathrm{~L} / \mathrm{min} / \mathrm{m}^{2}$. Dobutamine was injected at a rate of $5 \mathrm{mcg} / \mathrm{kg} / \mathrm{min}$. Despite dobutamine infusion, the heart rate decreased from 65 to $43 \mathrm{BPM}$, so $0.25 \mathrm{mg}$ of atropine was intravenously injected. As a result, heart rate increased to $100 \mathrm{BPM}$ and cardiac index increased to $3.9 \mathrm{~L} / \mathrm{min} / \mathrm{m}^{2}$. Dobutamine infusion was stopped after 10 minutes. Under TOF monitoring, Bridion was given at a dose of $4 \mathrm{mg} / \mathrm{kg}$, and the patient was extubated 20 minutes later.

\section{DISCUSSION}

Cardiomyopathy has become the leading cause of death in older DMD patients due to an improvement in respiratory therapy. Mechanical ventilation may also contribute to exacerbation of myocardial suppression by heart-lung interaction [5], so detailed cardiac function monitoring is required during operation. Cardiomyopathy associated with DMD usually begins at six years of age, and the incidence increases gradually with age. However, symptoms usually do not present below 10 years of age. Only $15 \%$ of patients under 14 years of age and $57 \%$ of those over 18 years manifest heart failure symptoms [6]. Therefore, it is difficult to diagnose cardiomyopathy in DMD patients. In this case, the patient was hospitalized because of recurring pneumonia without previous diagnosis of cardiomyopathy. Thinning of the left ventricle wall and progressive LV dilatation are poor prognostic factors [7]. In this patient, the diameter of the LV was increased (LVEDD/BSA $4.1 \mathrm{~cm} / \mathrm{m}^{2}$ ), but the wall did not show signs of thinning (septum $0.6-0.7 \mathrm{~cm} /$ posterior wall 0.7 $0.8 \mathrm{~cm})$.

Sinus tachycardia is the most common finding and is often accompanied by a shortened PR interval and right ventricular hypertrophy in ECG. Fragment QRS is also present in $83.7 \%$ of DMD patients, and this finding was associated with the degree of left ventricular function $[8,9]$. The patient in our case showed sinus tachycardia and fragmented QRS, but a normal PR interval on ECG. The cause and timing of sinus tachycardia development are not well known, but may be related to the onset of cardiomyopathy [10]. It is important to recognize baseline ECG abnormality as a predictor of disease progression. Common ECG abnormalities include a Q wave in lead III or V6, right ventricular hypertrophy, sinus tachycardia, tall right precordial R waves, and short PR interval [11].

Cardiac imaging is used to identify cardiomyopathy despite symptom absence. Cardiac index and hemodynamics are monitored in real-time, and treatment is performed accordingly. Patients with an ejection fraction less than 55\% may be considered for transesophageal echocardiography if major surgery is performed. Considering the reduction in systolic ability, it is helpful to increase the preload by the use of appropriate inotropes, such as dobutamine or milrinone. Measuring serum lactate to assess end organ perfusion is also helpful in therapeutic decisions.

Postoperative hemodynamic monitoring is necessary until respiration and fluid status are normalized. Echocardiography should be used to assess the left ventricular function and to treat cardiac rhythm abnormalities appropriately [12].

\section{CONCLUSION}

As the understanding of DMD pathophysiology increases, the incidence of complications related to anesthetic agents has decreased. However, cardiomyopathy has emerged as a new challenge during anesthesia. Cardiac status should be carefully evaluated preoperatively. Both the cardiologist and pulmonologist should coordinate to predict end organ involvement of DMD patients. The goal of anesthesia is to maintain end organ perfusion through proper functioning of the left ventricle. To achieve this goal, the anesthesiologist must pay careful attention to cardiac index, serum lactate measurement, and preload, along with proper usage of inotropes. 


\section{REFFERENCES}

1. Bushby K, Finkel R, "Diagnosis and management of Duchenne muscular dystrophy, part 1: diagnosis, and pharmacological and psychosocial management" Lancet Neurol. Vol.9, no.1: pp. 77-93, 2010.

2. Strehle E M, Straub V, "Recent advances in the management of Duchenne muscular dystrophy" Arch Dis Child. Vol.100, no.12: pp. 1173-1177, 2015.

3. Boivin A, Antonelli R, "Perioperative management of gastrostomy tube placement in Duchenne muscular dystrophy adolescent and young adult patients: A role for a perioperative surgical home" Paediatr Anaesth, Vol.28, no.2:pp. 127-133, 2017.

4. D'Amario D, Amodeo A, "A current approach to heart failure in Duchenne muscular dystrophy" Heart. Vol.103, no.22: pp. 1770-1779, 2017.

5. Mesquida J, Kim H K, "Effect of tidal volume, intrathoracic pressure, and cardiac contractility on variations in pulse pressure, stroke volume, and intrathoracic blood volume" Intensive Care Med. Vol.37, no.10: pp. 1672-1679, 2011.

6. Nigro G, Comi L I, "The incidence and evolution of cardiomyopathy in Duchenne muscular dystrophy" Int J Cardiol. Vol.26, no.3: pp. 271-277, 1990.

7. Connuck D M, Sleeper L A, "Characteristics and outcomes of cardiomyopathy in children with Duchenne or Becker muscular dystrophy: a comparative study from the Pediatric Cardiomyopathy Registry" Am Heart J. Vol.155, no.6: pp. 998-1005, 2008.

8. Bouhouch R, Elhouari T, "Management of cardiac involvement in neuromuscular diseases: review" Open Cardiovasc Med J. Vol.2: pp. 93-96, 2008.

9. Thrush P T, Allen H D, "Re-examination of the electrocardiogram in boys with Duchenne muscular dystrophy and correlation with its dilated cardiomyopathy" Am J Cardiol. Vol.103, no.2: pp. 262-265, 2009.

10. Thomas T O, Morgan T M, "Correlation of heart rate and cardiac dysfunction in Duchenne muscular dystrophy" Pediatr Cardiol. Vol.33, no.7: pp. 1175-1179, 2012.

11. Yoo $\mathrm{W} \mathrm{H}$, Cho M J, "The evolution of electrocardiographic changes in patients with Duchenne muscular dystrophies" Korean J Pediatr. Vol.60, no.6: pp. 196-201, 2017.

12. Cripe L H, Tobias J D, "Cardiac considerations in the operative management of the patient with Duchenne or Becker muscular dystrophy" Paediatr Anaesth. Vol.23, no.9: pp. 777-784, 2013. 